\title{
Atypical Phimosis Secondary to a Preputial Metastasis from Rectal Carcinoma
}

\author{
Maximilien C. Goris Gbenou ${ }^{a, c}$ Tawfik Wahidy ${ }^{a}$ \\ Karine Llinares ${ }^{b}$ Dominique Cracco $^{a} \quad$ Alain Perrot $^{a}$ \\ Dominique Riquet ${ }^{a}$ \\ Departments of ${ }^{a}$ Urology, and ${ }^{b}$ Histopathology, Centre Hospitalier de \\ Valenciennes, Valenciennes, and ${ }^{\mathrm{C}}$ Department of Urology, Centre Hospitalier \\ de Valence, Valence, France
}

\section{Key Words}

Colorectal cancer · Phimosis - Penile metastasis · Cutaneous metastasis

\begin{abstract}
Background: Cutaneous metastases from colorectal cancer are uncommon, accounting for $6.5 \%$ of all secondary skin lesions. They occur in advanced disease. The most common site is the abdomen. The penis is a rare site.
\end{abstract}

Case Report: We report the case of a 79-year-old patient who presented, two years after rectocolectomy for a rectal adenocarcinoma, obstructive renal failure secondary to a presacral recurrence, as well as symptomatic phimosis associated with papulonodules invading the penis, scrotum and pubis. After hemodialysis, percutaneous pyelostomy, and double-J catheter placement, the patient underwent circumcision and a pubic skin biopsy. On histology and immunohistochemistry, a cutaneous metastasis originating from the rectal adenocarcinoma was diagnosed.

Conclusions: Penile invasion by colorectal cancer revealed by phimosis is a rare occurrence but needs to be recognized and promptly diagnosed by biopsy in order to propose appropriate adjuvant therapy. These rare lesions can occur without any liver or lung involvement.

\section{Introduction}

Colorectal cancer most often disseminates to the liver and lungs via the lymphatic or hematogenous routes and rarely to the skin $[1,3]$. Colorectal cancer metastasizes to the skin in $6.5 \%$ of cases only, usually heralding generalized spread of disease [3]. Metastasis has been reported to abdominal surgical scars after colorectal tumor resection by laparotomy [11] or laparoscopy [10], to the head and neck, extremities, 
and penis $[4,5]$. Penile metastases affect the totality of the organ $[6,8]$. The foreskin is invaded by the same mechanism as other skin.

\section{Case Report}

A 79-year-old man was referred to our department for obstructive acute renal failure secondary to bilateral ureterohydronephrosis. The patient had a history of lower rectal adenocarcinoma, diagnosed three years earlier and treated by surgery. Recurrence occurred two years after surgery. Two presacral masses compressed both ureters with ureterohydronephrosis. Chemoradiation treatment resulted in a favorable clinical course, with regression of the dilatation of the left pyelocalyceal cavities.

During the 3 months prior to referral, the patient had suffered from extensive papulonodules (fig. 1) of the pubis, penis and scrotum, with severe penile edema and painful sclero-atrophic phimosis. Laboratory findings on admission signalled acute renal failure [creatine, $81 \mathrm{mg} / \mathrm{l}$ (normal = 8.4-12.5 $\mathrm{mg} / \mathrm{l}$ ); potassium, $8 \mathrm{mEq} / \mathrm{l}$ (normal = 3.5-4.5 mEq/l)]. Emergency hemodialysis was performed. A computerized tomography (CT) scan of the abdomen and pelvis revealed recurrence of bilateral dilatation of the pyelocalyceal cavities from the presacral masses.

Bilateral percutaneous pyelostomy improved renal function. Antegrade double-J catheters were placed. However, the patient experienced mounting voiding disorders affecting the skin lesions. Incomplete bladder drainage meant that the pyelostomy drainage tubes could not be removed. The high post-voiding residue increased bladder pressure, causing a vesicoureteral reflux on the double-J catheters and leakage around the pyelostomy tubes. Circumcision was performed to reduce pain and urinary disorders, and a biopsy of a pubic skin lesion was carried out (fig. 2). Histologically, it was a cutaneous metastasis of the rectal adenocarcinoma. It was positive for cytokeratin 20 on immunohistochemistry, which is a specific marker of colorectal tissue (fig. 3 ). The patient refused second-line chemotherapy. We opted for palliative care. The patient died 6 months after diagnosis of the cutaneous metastases.

\section{Discussion}

The overall incidence of cutaneous metastases, according to a meta-analysis, is $5.3 \%$ $[2,13]$. Cutaneous metastases from colorectal cancer are rare in the absence of hepatic lesions and usually occur when disease spread has generalized $[9,12]$. Our patient presented locoregional recurrence limited to the pelvis, without any signs of liver or lung metastases at the last radiographic assessment. On average, cutaneous metastases occur 4.9 years after colorectal cancer diagnosis but a 15 -year interval was noted in one case [3]. In our patient, they occurred after 36 months. Cutaneous metastases signal a poor prognosis. Mean survival after diagnosis is 3.3 months (range, 2-4.5) [3]. Several mechanisms may explain their occurrence: direct tumor extension, dissemination by the lymphatic and/or hematogenous routes, invasion of the ligaments of embryologic origin, or implantation of exfoliated tumor cells at surgical resection [14]. The presence of tumor emboli in the vessels, observed on histology (fig. 3), supports a lymphatic and/or hematogenous origin in our patient.

Invasion of the penis by colorectal cancer is rare and occurs on massive perineal extension of the tumor [15]. Invasion of the penis, scrotum and pubis from the foreskin as a result of atypical phimosis, as observed in our patient, seems to be extremely rare. Diagnosis necessitates a skin biopsy in order to identify the nature of the metastasis and to select appropriate management [7] (radiotherapy, chemotherapy, hyperthermia, or penectomy). Survival ranges from 3 to18 months $[3,15]$. Penectomy is a radical 
procedure, with a controversial survival benefit, to be reserved for penile metastases only [15]. In view of the extensive lesions in our patient, not amenable to surgery, and his refusal of second-line chemotherapy, we opted for palliative care.

In conclusion, the penis can be invaded by colorectal cancer from atypical phimosis. This lesion must be recognized early and diagnosed by biopsy in order to be able to propose appropriate adjuvant therapy. These rare lesions can occur in the absence of liver and lung metastases.

\section{Acknowledgements}

The authors thank Cendrine Halliez for help in correcting the draft report. The patient provided his consent to the writing of this report.

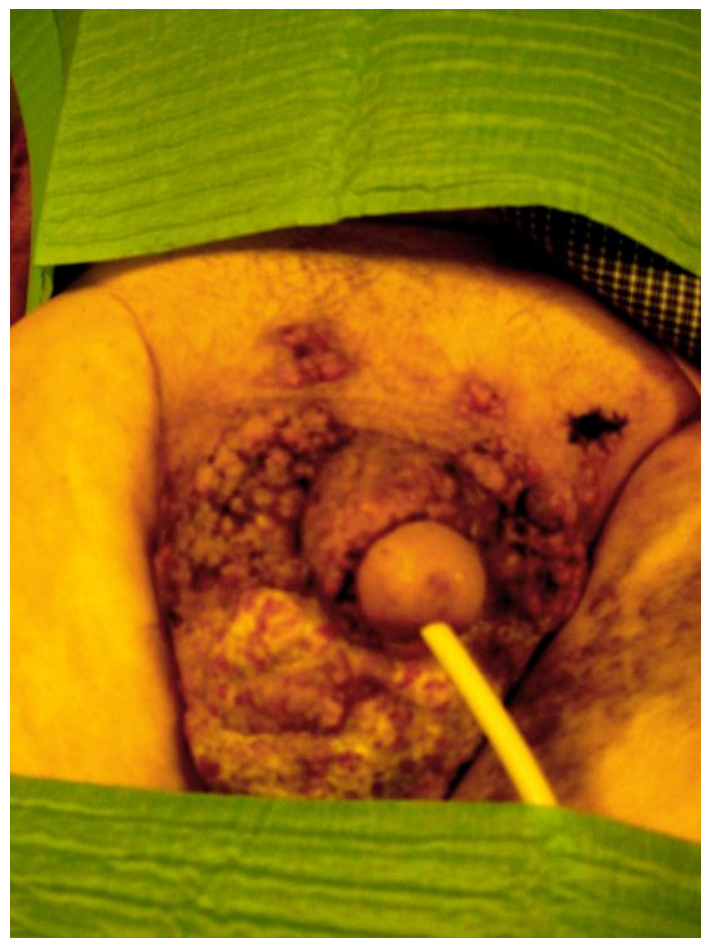

Fig. 1. Presence of papulonodules invading the pubis, penis and scrotum after circumcision and after insertion of bladder catheter. 


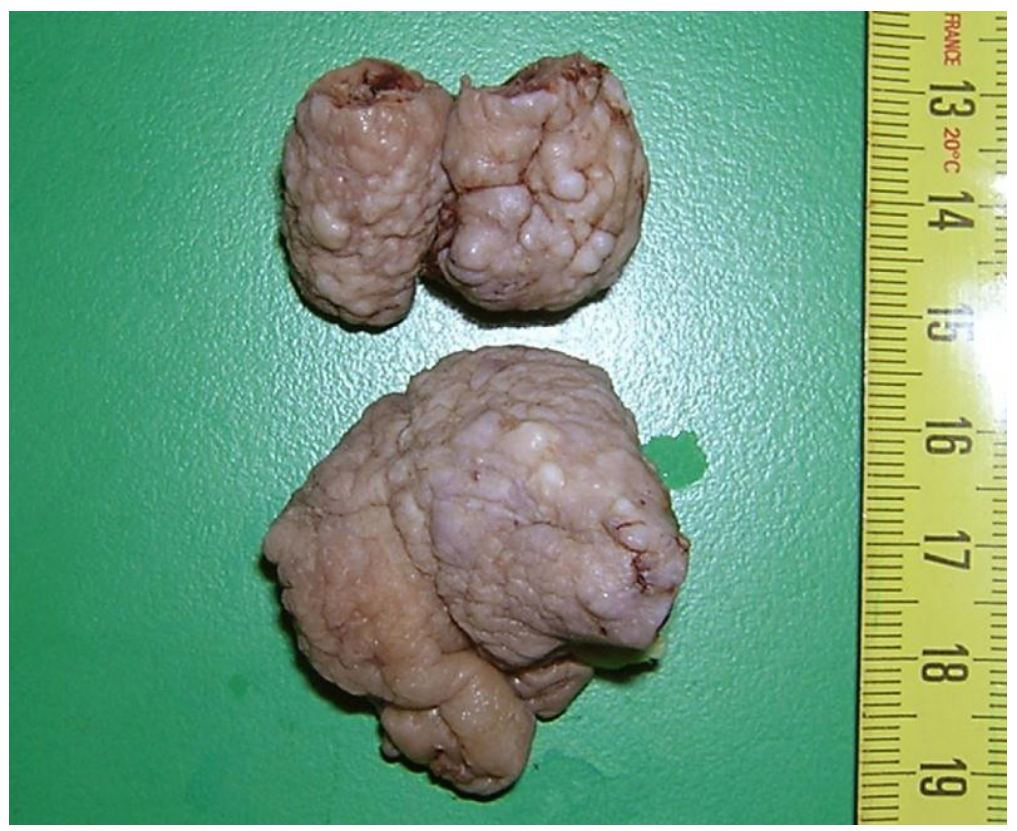

Fig. 2. Resected specimens of pubic skin (top) and foreskin (bottom) after fixing in formaldehyde.
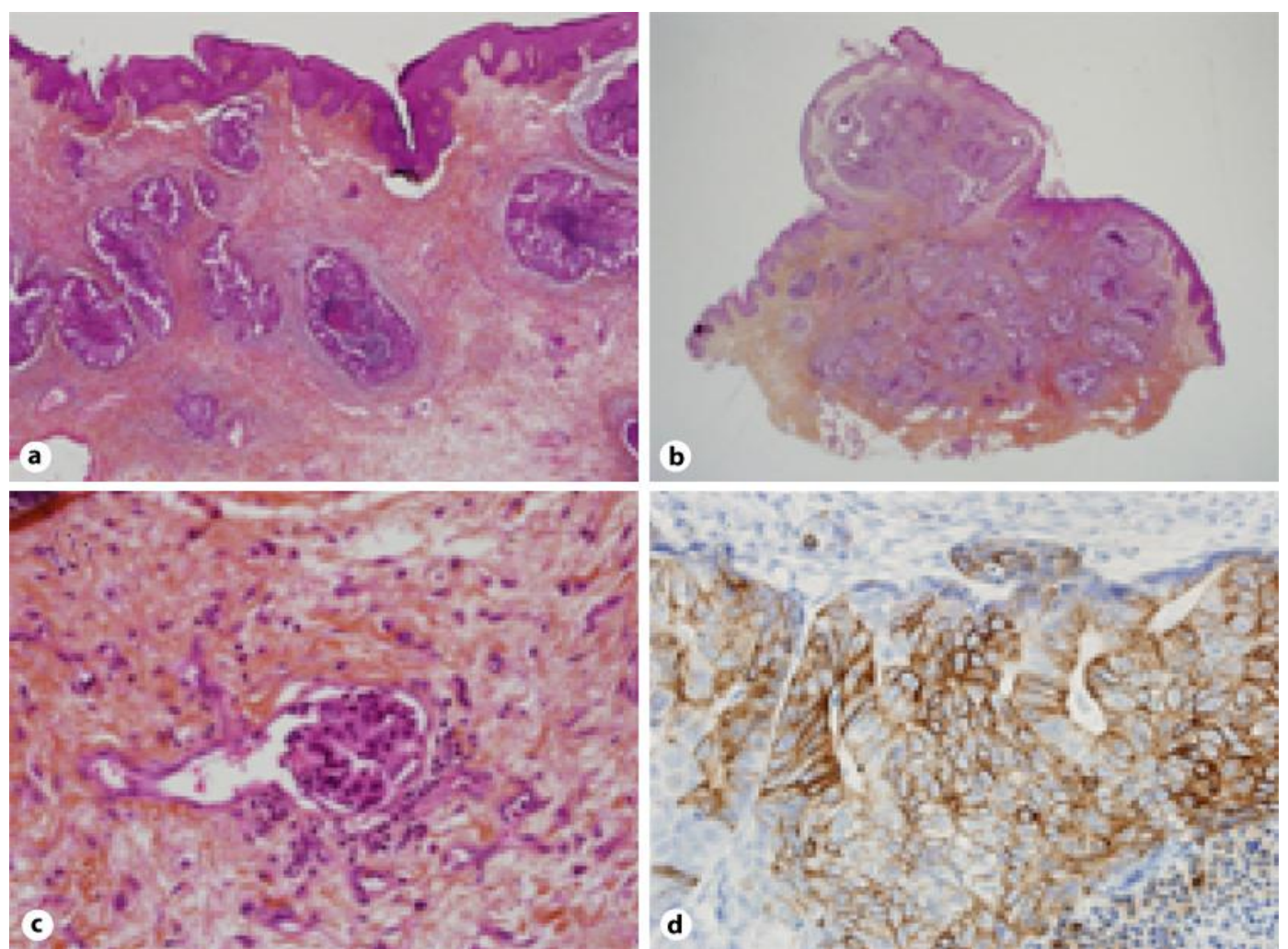

Fig. 3. Micrographs. a Foreskin invasion by rectal adenocarcinoma $(\times 40)$, b pubic skin biopsy showing rectal cancer $(\times 20)$, c venous emboli $(\times 400)$, d positive reaction to cytokeratin $20(\times 400)$. 


\section{References}

1 Sgambati SA, Barrows GH: Cutaneous metastasis of colon carcinoma: a case report. Conn Med 1993;57:665-667.

-2 Gottleid JA, Schermer DR: Cutaneous metastasis from carcinoma of the colon. JAMA 1970;213:2083.

-3 Saeed S, Keehn CA, Morgan MB: Cutaneous metastasis: a clinical, pathological, and immunohistochemical appraisal. J Cutan Pathol 2004;31:419-430.

4 Stavrianos SD, McLean RN, Kelly CG, Fellows S: Cutaneous metastasis to head and neck from colonic carcinoma. Eur J Surg Oncol 2000;26:518-519.

5 Banerjee GK, Lim KP, Cohen NP: Penile metastasis: an unusual presentation of metastatic colonic cancer. J R Coll Surg Edinb 2002;47:763-764.

6 Paquin AJ Jr, Roland SI: Secondary carcinoma of the penis: a review of the literature and a report of nine new cases. Cancer 1956;9:626-632.

7 Ben-Yosef R, Kapp DS: Cancer metastatic to the penis: treatment with hyperthermia and radiation therapy and review of the literature. J Urol 1992;148:67-71.

$>8$ Mukamel E, Farrer J, Smith RB, deKernion JB: Metastatic carcinoma to penis: when is total penectomy indicated. Urology 1987;29:15-18.

>9 Alexandrescu DT, Vaillant J, Yahr LJ, Kelemen P, Wiernik PH: Unusually large colon cancer cutaneous and subcutaneous metastases occurring in resection scars. Dermatology Online Journal 2005;11:22. http://dermatology.cdlib.org/112/.

10 Wright PK, Jha MK, Barrett PD, et al: Colonic adenocarcinoma presenting as a cutaneous metastasis in an old operative scar. J Postgrad Med 2003;49:157-158.

11 Iwase K, Takenaka H, Oshima S, et al: The solitary cutaneous metastasis of asymptomatic colon cancer to an operative scar. Surg Today 1993;23:164-166.

12 Morton BA, Scholes J, Kral JG: An unusual presentation of colon cancer. J Surg Oncol 1986;33:92-94.

13 Krathen RA, Orengo IF, Rosen T: Cutaneous metastasis: a meta-analysis of data. South Med J 2003;96:164-167.

-14 Reilly WT, Nelson H, Schroeder G, Wieand HS, Bolton J, O’Connell J: Wound recurrence following conventional treatment for colorectal cancer. A rare but perhaps underestimated problem. Dis Colon Rectum 1996;39:200-207.

15 Lo S, Crew J: Penile metastasis from rectal carcinoma. Singapore Med J 2004;45:299. 